University of Warwick institutional repository: http://go.warwick.ac.uk/wrap This paper is made available online in accordance with publisher policies. Please scroll down to view the document itself. Please refer to the repository record for this item and our policy information available from the repository home page for further information.

To see the final version of this paper please visit the publisher's website. Access to the published version may require a subscription.

Author(s): Adelman, James S and Brown, Gordon D. A.

Article Title: Modeling lexical decision: The form of frequency and diversity effects.

Year of publication: 2008

Link to published version:http://dx.doi.org/ 10.1037/0033-

295X.115.1.214

Publisher statement: 'This article may not exactly replicate the final version published in the APA journal. It is not the copy of record.' 
Adelman, J. S., \& Brown, G. D. A. Modeling lexical decision: The form of frequency and diversity effects. and Postscript: Deviations from the predictions of serial search.

DOI: 10.1037/0033-295X.115.1.214

In press, Psychological Review, as at October 24, 2007. This article may not exactly replicate the final version published in the APA journal. It is not the copy of record. 
Running head: MODELING LEXICAL DECISION

Modeling Lexical Decision: The Form of Frequency and Diversity Effects

\author{
James S. Adelman and Gordon D. A. Brown
}

University of Warwick

Corresponding Author:

James S. Adelman

Department of Psychology,

University of Warwick,

Gibbet Hill Road,

COVENTRY,

CV4 7AL,

UK.

Telephone: +44 (0) 2476150233

Electronic mail: J.S.Adelman@warwick.ac.uk 


\begin{abstract}
What is the root cause of word frequency effects on lexical decision times? Murray and Forster (2004) argued that such effects are linear in rank frequency, consistent with a serial search model of lexical access. This paper (i) describes a method of testing models of such effects that takes into account the possibility of parametric overfitting; (ii) illustrates the effect of corpus choice on estimates of rank frequency; (iii) gives derivations of nine functional forms as predictions of models of lexical decision; (iv) details the assessment of these models and the rank model against existing data regarding the functional form of frequency effects; and (v) reports further assessments using contextual diversity, a factor confounded with word frequency. The relationship between the occurrence distribution of words and lexical decision latencies to those words does not appear compatible with the rank hypothesis, undermining the case for serial search models of lexical access. Three transformations of contextual diversity based on extensions of instance models do however remain as plausible explanations of the effect.
\end{abstract}




\section{Modeling Lexical Decision: The Form of Frequency and Diversity Effects}

The effect of word frequency on lexical processes is both ubiquitous and large. It is evident in a wide range of both tasks (e.g., lexical decision, word naming, picture naming and memorial tasks) and measures (especially response times and eye fixation times, but also errors in the aforementioned tasks). It therefore seems reasonable to require models of lexical tasks to account not only for the existence of frequency effects, but also for their form. However, few existing models provide such an account. Murray and Forster (2004) took up the challenge in the context of lexical decision times. Specifically, they showed that their bin model, a frequency-ordered serial-search model of lexical access, predicts that lexical decision times will be determined by the rank ordering of word frequency. In comparisons between rank and logarithmic transformations, they argued that just this pattern could be seen in the data, and (inter alia) interpreted this result as evidence for serial search models.

As Murray and Forster (2004) made clear, one advantage of a clear theoretical framework, such as their serial search model, is that it gives a principled prediction of the functional form of frequency effects. According to Murray and Forster's bin model, tasks such as lexical decision require serial search of frequency-ordered lists. The expected ranked position of a word in any list is a linear function of the word's rank frequency, leading to the prediction that expected search time will be linear in rank word frequency. We agree with Murray and Forster that future progress will require the derivation and test of specific predictions of fully explicated models such as the one described in their paper. In particular, the evaluation of whether the ranked frequency of a word, rather than some other (e.g., logarithmic, power, or exponential) transformation of frequency, predicts lexical decision time would appear to have wide implications for quite general classes of lexical access model. 
In this paper we consider several different classes of model architecture and derive their specific predictions regarding the functional form of the word frequency effect (e.g., logarithmic, power and exponential from Bayesian, instance and contextual cue models respectively). This allows us to evaluate different classes of model against several large data sets. In particular, we explore some of the specific claims made by Murray and Forster (2004) and question their conclusions regarding serial search models, before turning to a more general survey of predictions regarding lexical decision latencies.

The plan of the paper is as follows. First, we examine Murray and Forster's dismissal (on the grounds of complexity) of the power transformation. We show that additional tests on Murray and Forster's own data suggest the superior fit of the power transformation is not solely due to its propensity to overfit due to complexity. Second, we argue (contrary to Murray and Forster's assumption) that high correlations in frequency among corpora do not guarantee accurate assessment of models based on rank word frequency, suggesting the use of alternatives to the Kučera and Francis (1967) word frequency counts. In the third part of the paper, we derive predictions concerning the functional form of word frequency effects from several different classes of model. In the fourth part of the paper, we test these predictions regarding frequency effects with large data sets and find evidence against a number of models. Finally, we argue that it is not word frequency but rather the correlated factor of contextual diversity that determines latency of access (McDonald \& Shillcock, 2001; Adelman, Brown, \& Quesada, 2006), and conduct similar analyses with this variable. Overall, the additional analyses that we report narrow the model types that can be considered compatible with existing data.

The evidence adduced by Murray and Forster (2004) for their model was based on three new lexical decision experiments and on analyses of a subset of the data from a mega-study by Balota, Cortese, and Pilotti (1999), using only the Kučera and Francis (1967) frequency count. The largest difference in prediction of response times (favoring 
rank frequency over log frequency) was obtained for the Balota et al. (1999) data. Murray and Forster (2004) used only lexical decision data to support their claims regarding lexical access; we also consider only data from this task. Additional assumptions allowed an extended version of the bin model to account for error rates (and error response times), but the signature effect of such a model is a linear relationship between rank frequency and (correct) lexical decision times, so any case against the model is both sufficient and strongest if it is based on the response times. In addition, a focus on latencies rather than error rates allows straightforward derivation of predictions from other classes of model, and more accurate assessment of such predictions (because variance in error rates is high, especially at the item level).

\section{The Methodology of Comparing Fits of Frequency}

\section{Transformations}

We first consider two methodological aspects of the Murray and Forster's (2004) assessment of transformations of frequency. These give the motivation for some of our additional analyses.

\section{Comparing Rank and Power Law Fits}

Murray and Forster (2004) found that a power law transformation of frequency gave a higher $R^{2}$ on their (Experiment 1 and 2) data than did the rank of frequency. However, they suggested that this result might have been an artifact arising from the additional parameter of the power function. They supported this claim by simulations, the results of which illustrated the fact that $R^{2}$ values can misleadingly favor a two-parameter power function over a one-parameter linear function ${ }^{1}$.

This observation does not itself, however, indicate that the power law functional form should be dismissed. It is important to know whether the observed $R^{2}$ advantage for the more complex function is of about the expected size on the basis of this bias, or is 
larger than expected, as only the latter gives evidence against the simpler function. To discover this, one can instead simulate many sets of data under the hypothesis that the rank function generates response times in the task, in order to estimate the true distribution of the power law $R^{2}$ (or any other statistic) under the rank hypothesis, and then test whether the observed value falls in the tail of this distribution. In order to generate response times under the rank (or any other) hypothesis, it is necessary to make assumptions about the distribution of response times, because typically models predict central tendency (in this case mean lexical decision time), but not the dispersion around that central tendency. When a hypothesis is instantiated by using the empirical (observed) distribution to estimate the distribution of the population under that hypothesis, and the distribution of a statistic is examined under repeated resampling (with replacement, and especially, but not necessarily, by simulation), this is known as a bootstrap (Efron \& Tibshirani, 1993).

Within the data we used here, the predictors, including frequency (however transformed), are effectively fixed factors ${ }^{2}$. The appropriate procedure is then as follows. One first fits the model under test $\left(H_{0}\right)$. Estimates are thereby obtained of the model's parameters, and of the distribution of errors in prediction (i.e., residuals). The observed error distribution estimate is then used $^{3}$ to generate simulated data around the predictions of the model with the estimated parameters. This step is repeated many times to produce several $(B)$ bootstrap 'replications' of the experiment of interest. For each of these, some statistic $(T)$ of interest (for which higher values indicate more $H_{0}$ misfit) has an estimate $\left(t_{i}^{*}\right)$ calculated. These $t_{i}^{*}$ values form an estimate of the overall distribution of this statistic, which can be compared with its observed value $(t)$ from the genuine experiment. The size of the tail this cuts off $\left(\hat{p}=\#\left(t^{*}>t\right) / B\right.$ for $T$, where low $\hat{p}$ values are evidence against $H_{0}$ ) can be used for a (usually one-tailed) null-hypothesis significance test, with results in the tail taken as cause to reject the model under test. The tests we 
discuss in this paper use the fit of some other model $\left(H_{a}\right)$ as a component of the statistic $T$ (most simply, the $R^{2}$ for $H_{a}$ ) so that evidence against $H_{0}$ is based on systematic deviations from its predictions.

In our first illustrative analysis, we conducted simulations with the rank model as $H_{0}$ and examined the $R^{2}$ of a power-law based model acting as $H_{a}$. (The derivation of such a model with the exponent as a free parameter is given later in the paper.) Figure 1 shows the estimated distribution of a power function's $R^{2}$ (this acting as $T$ ), when rank frequency generated the simulated data, with the simulation constructed on the basis of the condition means from Murray and Forster's (2004) Experiment 1. The mean was $90.3 \%$, similar to the value obtained by Murray and Forster in their higher variance set of simulations, and higher than the baseline (88.4\%) for the fit of rank. This result is consistent with the assertion that the $R^{2}$ estimate is very biased. The observed $R^{2}$ for the power law functional form was $92.1 \%$. This value did not fall in the $5 \%$ tail of the distribution simulated under the rank hypothesis; in fact, $\hat{p}=.324$. However, whether the $R^{2}$ of the power function is surprisingly high (given the rank hypothesis is $H_{0}$ ) is better considered by comparing it to the baseline $R^{2}$ of the rank function, and asking whether the difference is surprisingly high. This procedure ameliorates the influence of idiosyncratic properties of the sample. In many of the runs of the simulation (or indeed actual replications of the experiment) where the power $R^{2}$ is high, the rank $R^{2}$ will also be high, as the predictions of the two models are in many cases very similar. For instance, both will obtain their better fits when the frequency to response time relationship is monotonic. Failing to take account of this relationship between the two values of $R^{2}$ being compared therefore reduces the power of the test, just as using a between-subjects $t$-test loses power when a within-subjects $t$-test is appropriate. Thus, some paired comparison is necessary. The simplest such method, illustrated in Figure 2, uses the difference score of the $R^{2}$ values as the statistic of interest $(T)$. The distribution of this statistic had a 
positive mean, again pointing to bias, but the observed difference of $3.7 \%$ was now in the $5 \%$ tail $(\hat{p}=.024)$. This provides evidence that the extent to which the $R^{2}$ was higher for a power-law function cannot be explained away by the tendency for power laws to fit data generated according to the rank hypothesis better than rank frequency ${ }^{4}$. Such a result appears to be inconsistent with the serial search hypothesis.

There are two ways, however, in which such a methodology is incomplete. First, it may depend too heavily on the specific parameters that are estimated. This type of simulation treats these parameter estimates as exact, generating a distribution (of $T$ ) that gives the correct rejection rate only if the estimates obtained are without error. The standard $t$-test resolves this problem with respect to variance when comparing two means; dividing through by the standard error creates a statistic whose distribution is independent of the variance. We used a technique known as the double bootstrap (whose details are described by Beran, 1988 $)^{5}$ to find a statistic that had the property of being relatively independent of the parameter estimates in exploratory simulations. Omitting the details, the statistic used in the Hotelling $t$-test for comparing $r$ values appeared satisfactory (although its distribution was not the Student's $t$ that would occur for the hypothesis that the $r$ values are equal, as is usually tested with this statistic). A single bootstrap simulation of the Murray and Forster's (2004) Experiment 1 (condition mean) data with this statistic, under the rank hypothesis, is given in Figure 3. Again, the rank hypothesis was rejected $(\hat{p}=.048)$.

Second, no examination has been made of whether the rank model can provide evidence against the power model; since the models are not nested, the rank model can fit better than one might expect were the power model true. We might find that both models fit better than would be the case if the other were true if some hybrid model, some mixture model, or some unrelated third model were in fact generating the data. Figure 4, generated under a power model hypothesis, illustrates that such a possibility does not 
appear to be the case with these data: The power model was not rejected $(\hat{p}=.402)$. Notably, were the empirical fits of the two models equal, the result would have constituted evidence against the power model (with $\hat{p}=.008$ ); the implication is that the rank model could in principle have given cause to reject the power model even when its $R^{2}$ was smaller than the power law's. However, it was sufficiently inferior that in this analysis it did not give evidence against the power model. Hereafter, in presenting Monte Carlo bootstraps, we will report only $\hat{p}$ values, without plotting the full distribution.

\section{The Distortion of Rank Frequency}

The analyses above are consistent with a power law generating response times in lexical decision, but not with rank doing so, giving evidence against serial search models. A source of caution about these results and those of Murray and Forster (2004) is the extent to which it matters which corpus is counted to derive the estimate of word frequency. Murray and Forster noted that their data might be criticized on the basis of their use of the Kučera and Francis (1967) frequency count, as there are counts based on larger corpora, and by comparison, the accuracy of the Kučera and Francis estimates of frequency for low-frequency words may be limited. To counter this possibility, Murray and Forster noted the high (.900) correlation between the Kučera and Francis frequencies and CELEX (Baayen, Piepenbrock, \& Gulikers, 1995) frequencies for their Experiment 2 items, and argued that the counts are therefore so similar that the possible discrepancies at the low-frequency end of the scale are too small to be of concern.

Murray and Forster (2004) also noted that lexical decision latencies from their Experiment 2 correlated similarly with log frequency as derived from Kučera and Francis (1967) and CELEX. However, the $R^{2}$ of rank Kučera-Francis frequency in accounting for these latencies was similar to that of log Kučera and Francis frequency, and so a more accurate measure of rank frequency might correlate less well with lexical decision time. If 
so, evidence in favor or against models making the rank prediction could be spurious. More importantly, other larger counts might improve the correlation among the variables. Therefore we conducted analyses using frequency counts derived from (1) Kučera and Francis; (2) the 12th grade level (and below) texts ${ }^{6}$ of the LSA TASA corpus (Landauer, Foltz, \& Laham, 1998); (3) CELEX; and (4) the BNC (British National Corpus Consortium, 2000). Use of these corpora allowed us to construct frequency counts based on $1.0,8.3,17.9$, and 84.5 million word tokens respectively.

As Murray and Forster (2004) noted, when calculating ranks of frequencies, it is important to take account of the fact that the corpus will probably contain words unknown to the participant pool, and which therefore will not be searched (assuming a rank model is true). For all the corpora, we calculated adjustments to estimates of rank in a fashion similar to Murray and Forster. A total of 30,196 types chosen from the corpora randomly were each checked in an unspeeded fashion by one of two undergraduate raters for whether they were known. For each frequency in each corpus, the proportion of unknown words was used to adjust the rank of words of lower frequency and words of the same frequency (to obtain the correct tied rank); for the higher frequency words, adjacent frequencies were merged into bins due to few observations.

Comparison of the relationships among rank frequencies in Figure 5a demonstrated that the ranks of many words in the relatively small Kučera and Francis (1967) counts will be underestimated quite greatly at the low frequency end of the scale, and that this distortion is not linear, meaning that predictions generated linearly from these estimates will not correctly reflect a rank model. This occurs because some words of moderately low frequency are missing from the corpus, permitting words of lower frequency that are in the corpus to leapfrog them, without the higher frequency words occuring to balance the error (for instance, Burgess \& Livesay, 1998, listed CUCUMBER, DIPLOMA and REPTILE among the words missing from the Kučera-Francis counts). By contrast, in Figure 5b log 
frequency can be seen to be only slightly distorted, because for word frequency (unlike rank) the main source of distortion is a few words whose genuine frequency is just below one per corpus size.

Some frequency counts are more reliable and predictive than others (e.g., Burgess \& Livesay, 1998; Balota, Cortese, Sergent-Marshall, Spieler, \& Yap, 2004). Moreover, there are systematic and distortive differences in estimates of rank frequency. It seems possible that the distortion in the estimation of rank frequency might account for its apparent superiority, especially as the predicted response times from rank for the lowest frequency words already were too long, and correcting the distortion can only exacerbate this problem.

There are two ways that have been described in which the methodology of assessment of transformations of word frequency can be extended. First, bootstrap tests can be used that are based on testing the hypothesis that a model is correct, allowing a strict test that is suitable for models with several parameters. Second, more accurate estimates of frequency, and (consequently) less biased estimates of rank frequency, can be used in these assessments. Although we have adduced some evidence in favor of the power model and against rank, the analyses were made with a small frequency count that will have distorted predictions with respect to rank. Before turning to our full analyses of several data sets, we consider the predictions of other models of lexical decision with respect to the functional form of word frequency effects.

\section{Alternatives to the Rank Model}

With the machinery in place to test functional forms of frequency effects, the next two sections of this paper (i) derive the predictions of several classes of model for this functional form (e.g., logarithmic and power for Bayesian and instance models, respectively), and (ii) test these predictions against large data sets using the bootstrap 
methodology.

\section{Bayesian Model}

The Bayesian Reader model (Norris, 2006) is based on the assumption that the decision processes involved in visual word recognition tasks are based on a criterion for posterior probability of stimuli under priors based on word frequency. A simplified version of the Bayesian Reader predicts a logarithmic relationship between frequency and lexical decision latencies as we now show. Suppose for a given input word $w$ among all words $W$, the probability density of a particular input pattern $x_{t}$ occuring on any time tick $t$ is $f_{w}\left(x_{t}\right)$, and that when a nonword is presented the probability of a given input pattern $x_{t}$ on any given time tick is $g\left(x_{t}\right) . \pi_{w}$ denotes the prior for a word $w$. Then a Bayesian model stops and responds correctly for a word at the smallest $T$ such that the posterior probability of the input being a word exceeds a threshold $\tau<1$. That is:

$$
\frac{\sum_{w \in W} \pi_{w} \prod_{t=1}^{T} f_{w}\left(x_{t}\right)}{\sum_{w \in W} \pi_{w} \prod_{t=1}^{T} f_{w}\left(x_{t}\right)+\prod_{t=1}^{T} g\left(x_{t}\right)}>\tau .
$$

If the inputs come from a particular word $w$, then the $x_{t}$ come from the distribution defined by $f_{w}$. If we neglect the contribution from the other words (neighborhood effects), then the rule is:

$$
\frac{\pi_{w} \prod_{t=1}^{T} f_{w}\left(x_{t}\right)}{\pi_{w} \prod_{t=1}^{T} f_{w}\left(x_{t}\right)+\prod_{t=1}^{T} g\left(x_{t}\right)}>\tau,
$$

which is equivalent to

$$
\frac{\pi_{w} \prod_{t=1}^{T} f_{w}\left(x_{t}\right)+\prod_{t=1}^{T} g\left(x_{t}\right)}{\pi_{w} \prod_{t=1}^{T} f_{w}\left(x_{t}\right)}<\frac{1}{\tau},
$$


since probability densities and priors are positive. This is the same as:

$$
\frac{\prod_{t=1}^{T} g\left(x_{t}\right)}{\pi_{w} \prod_{t=1}^{T} f_{w}\left(x_{t}\right)}<\frac{1-\tau}{\tau} .
$$

Taking logarithms and switching signs,

$$
\log \pi_{w}+\sum_{t=1}^{T}\left(\log f_{w}\left(x_{t}\right)-\log g\left(x_{t}\right)\right)>\log \tau-\log (1-\tau) .
$$

Under independence of the $x_{t}$, this is a random walk with threshold $\log \tau-\log (1-\tau)$, mean drift $E_{f_{w}}\left(\log f_{w}\left(x_{t}\right)-\log g\left(x_{t}\right)\right)$, and starting point $\log \pi_{w}$. Since the drift is on average linear, the expectation of the stopping time (smallest $t$ ) is (negative) linear in the starting point $\log \pi_{w}$. If the priors are based on word frequency then this model will predict that a logarithmic functional form in frequency will dominate lexical decision latencies. Notably, the predicted response times do not depend on the total number of word experiences (summed subjective frequency over the whole vocabulary) because proper priors sum to one.

\section{Instance Model}

We now show that instance models predict various types of power law, depending on the details of their instantiation. Suppose that each experience with a word leaves an instance (trace) in memory, following the instance model of automatization (Logan, 1988). (This model is not the same as Logan's in that there is no algorithmic component.) Upon later presentation of that word in the lexical decision task, the time taken to retrieve each trace follows a Weibull distribution, whose cumulative distribution function $F(x)=1-e^{-x^{\alpha}}$ with $\alpha>0$ a free parameter. A positive lexical decision is made when the first such trace is retrieved, with density $L_{n}$ depending on the number of traces $n$, thus (Colonius, 1993):

$$
L_{n}(x)=1-(1-F(x))^{n}
$$




$$
\begin{aligned}
& =1-\left(e^{-x^{\alpha}}\right)^{n} \\
& =1-e^{-n x^{\alpha}} \\
& =1-e^{-\left(n^{1 / \alpha} x\right)^{\alpha}}
\end{aligned}
$$

and since the $x$ values are scaled by $n^{1 / \alpha}$ and $\alpha$ and $n$ are constants with respect to $x$, the expectation of this is proportional to $n^{-1 / \alpha}$ giving a power law functional form in frequency (as number of experiences is proportional to frequency).

Extension with Background Instances. If $b$ further instances representing the nonword alternatives or other, similar, words race with those representing the presented word, then the expectation of response time is proportional to $(b+n)^{-1 / \alpha}$, the generalized power model. If the exponential distribution, that is Weibull with $\alpha=1$, is used, then expected response times are $1 /(b+n)$, a more common race model.

\section{Duration-Practice Model}

Next, we consider a model in which decision times reduce in direct proportion to practice. It is simple to show that such a model predicts an exponential function form of frequency effects. For notation, assume that on the $(n+1)$ th experience (after $n$ previous experiences) with a word it takes $T_{n}$ to complete the access component of the lexical decision (and some additional period of time for residual (perceptual, motor) components). Suppose that on the $n$th occasion, practice occurs to a factor $0<\theta<1$, such that the time taken is reduced by $\theta T_{n-1}$ for the next occasion. That is, $T_{n}=T_{n-1}-\theta \cdot T_{n-1}=(1-\theta) T_{n-1}$. Clearly then, $T_{n}=(1-\theta)^{n} T_{0}=\exp (n \cdot \log (1-\theta)) T_{0}$, giving an exponential function in frequency (Heathcote, Brown, \& Mewhort, 2002).

\section{Restricted Contexts Model}

We now consider a model in which word frequency effects occur because frequent words are more likely to have occurred in a context that overlaps in representation with 
the context at test; such a model also predicts an exponential functional form. Suppose that $m$ distinct contexts can be mentally represented, and each occurrence of a word occurs in each of these contexts with equal probability. After $n$ occurrences of a word, given an arbitrary (random) new experimental context, the probability that it has not been seen before in this context is given by $\left(\frac{m-1}{m}\right)^{n}$ (the probability that it occurred $n$ times in one of the other contexts), and this is equal to $\exp (n \cdot \log ((m-1) / m))$. If words not seen in the current context on average generate slower lexical decisions (but response times are not otherwise conditional on frequency), then this again gives an exponential function in frequency.

\section{Hybrid Context-Instance Model}

More complex models can be considered. The above model can be augmented with the assumption that if the word has not been seen in the present context, all the other contextual instances race, as in the Instance model, to be retrieved. The overall expected response time will then be the product of the exponential function and the (generalized) power function giving an (A)PEX function (Heathcote et al., 2002).

\section{An Interactive-Activation model}

Finally, we consider an interactive-activation (IA) model that is similar in operation (with squashing action removed) to the lexical route of the DRC (Coltheart, Rastle, Perry, Langdon, \& Ziegler, 2001) with only a specific lexical activation (not a total lexical activation) rule. The external input to a word unit is constant when the activation of the letter units is constant, which typically occurs very early in processing in the DRC, so we treat it as a constant. There is additional (negative) input to the word unit, which represents an influence linear in some transformation $g$ of frequency, so overall the input is 
of the form $j+k g$. The change in activation from one time step to the next is:

$$
a_{t+1}-a_{t}=j+k g-\theta a_{t}
$$

Approximating this as a continuous process gives:

$$
\frac{d a}{d t}=j+k g-\theta a
$$

whose solution for the time $T$ at which activation reaches a threshold $A$ is

$$
T=\frac{1}{\theta} \log \left(\frac{j+k g}{j+k g-\theta A}\right)
$$

an inverse logistic equation, which is proportional to $\log ((a+g) /(b+g))$. In the DRC, $g$ is the logarithm of frequency (hereafter IA2), but a plausible alternative is to have $g$ directly proportional to frequency (hereafter IA1).

\section{Recapitulation}

There are several alternative models that make predictions as to the functional form of the word frequency effect. Those derived in this paper are as follows. A Bayesian model predicts a logarithmic relationship between frequency and response times. An instance model predicts a simple power law with negative exponent. An extended instance model with background elements predicts an adjusted (generalized) power relationship, where a constant is added to the frequency before before the power law is applied. A race model is a special case of this model with the exponent fixed as -1. Duration-practice or restricted contextual representation models predict an exponential relationship. Hybrid context-instance models make predictions that are those of the two models multiplied together (PEX from power or APEX from adjusted power). Interactive activation models predict an inverse logistic function in frequency (IA1) when they have bias linear in frequency, and an inverse logistic function in log. frequency (IA2) when they have bias linear in log. frequency. 


\section{Frequency Counts and Fits}

We have shown that there are several theoretically plausible functional forms of the relationship between word frequency and lexical decision latencies in addition to the rank hypothesis considered by Murray and Forster (2004). This section of the paper uses the bootstrap procedure previously described to examine the evidence for the rank and other models that is given by data in Murray and Forster's Experiment 1, and mega-studies conducted by Balota et al. (1999) and Balota et al. (2002, 2007). In the light of the differences we have detailed that may arise between frequency counts, we used all four frequency counts that we have already considered above.

We first considered the data of Murray and Forster's (2004) Experiment 1. 471 of the $474^{7}$ items used in that experiment appear in all four corpora. Although these data were collected to fill frequency conditions or bands (as they cover a small range of frequencies), it would be inappropriate to conduct tests using bands for the frequency counts not used to design the experiment, so all our analyses are based on items. We also considered (raw) mean response time data from three mega-studies, young and older adult samples reported by Balota et al. (1999), and the Elexicon (Balota et al., 2002, 2007) database. For these, we included as covariates the length (in letters), the orthographic neighborhood size (Coltheart, Davelaar, Jonasson, \& Besner, 1977; Andrews, 1989, 1992, as this variable correlates with frequency and affects lexical decision), and the number of syllables. We also included all the available $\mathrm{data}^{8}$, not only those in a restricted frequency range: As Murray and Forster noted in the rationale of their Experiment 2, the distinction between transformations of frequency will be most evident at the extremes of the frequency range. The range examined here is approximately .01 to 65000 per million (a ratio more than four orders of magnitude greater than was the case in Murray and Forster's Experiment 2) when the largest corpus is considered

As a summary measure, the $R^{2}$ value is reported for each combination of data set 
and transformation (with optimized parameters) in Table $1^{9}$. Many of the other transformations of frequency exhibited a higher $R^{2}$ than rank on most of the dataset-corpus combinations. Notably, with the data of Murray and Forster's (2004) Experiment 1, an advantage obtained for rank over log only when Kučera and Francis (1967) was used.

Of course, simply observing that a particular $R^{2}$ value exceeds another is not a valid statistical reason for rejecting a particular model; rejection should occur when it is implausible that the model produced the data. Table 2 therefore presents $\hat{p}$ values from bootstrap analyses of the rank model and the various other models, using the procedure detailed earlier using the Hotelling statistic on the $r$ values from the two models, treating the rank model as the model under test (i.e., the simulated model, $H_{0}$, against which these are one-tailed tests $)^{10}$. Therefore each value indicates the extent to which the results are surprising under the rank model, with low values indicating unexpectedly good fit for the alternative model. (The measurement of unexpectedness is under the assumption that the rank model is true, so high $\hat{p}$ values are not evidence against the alternative; separate tests, described below, are needed for this.)

In the vast majority of cases (117/144, including every test for the Elexicon data), the alternative model fitted significantly better than it would were word frequency effects in lexical decision governed by rank of frequency ${ }^{11}$. In the interest of giving the model under test the benefit of the doubt, in our interpretation we gave precedence to the test performed with the highest $R^{2}$ for the model under test $\left(H_{0}\right.$, i.e., rank). In 33 out of 36 such comparisons, the evidence gave a result significant against rank at the $5 \%$ level. In some of these cases there was evidence against the rank model even when the alternative model had a lower $R^{2}$ : For instance, log frequency would not have fared nearly so well as it did had rank produced the data, even in many cases where it appeared inferior. Critically, this means that there is systematic variability in the response times that can be 
attributed to word frequency but for which rank frequency cannot account. In each case, the conclusion is not that the alternative model is correct. In particular, the results do not license an argument in favor of the log transformation; if a log transformation were generating the data, it would have consistently had a higher $R^{2}$ value than rank (because their complexity is equivalent). Evidence has also been found against a log transformation previously by Balota et al. (2004), whose analyses found a quadratic tendency in log frequency to be significant (albeit before taking into account covariates) in the data of Balota et al. (1999).

To determine which, if any, of these other models were plausible in the light of the level of rank's fit to the data, tests swapping the roles of the models (i.e., switching $H_{0}$ with $H_{a}$ ) are presented in Table 3 . Each value indicates the extent to which the results are surprising under the particular $\left(H_{0}\right)$ model, with low values indicating unexpectedly good fit for the rank model, giving evidence against the $H_{0}$ model. As has already been related, the logarithmic frequency model could also be rejected; 14 out of 16 comparisons were significant (at a $5 \%$ level), and for three data sets, this included that with the corpus most favorable to $\log$ in terms of $R^{2}$. 10 out of 16 comparisons were disfavorable to the power transformation, all on the mega-study data; for all of the mega-studies, a highly significant result occurred for the most favorable corpus. 6 out of 16 comparisons gave evidence against the race model, including with the three larger corpora on Elexicon. 13 out of 16 comparisons gave significant results against the exponential model, including all comparisons with older adults, and all with Elexicon. Both the power-exponential (PEX) and IA1 models had 3 out of 16 results significant against them, including a highly significant result in the Elexicon-BNC combination, and the BNC performed best on Elexicon for this hypothesis. The situation with generalized power was less clear. Although 3 out of 16 results went against it, including the that with Elexicon-BNC combination, this result was not highly significant $(\hat{p}=.047)$; and the one highly 
significant result was with TASA on the young adult monosyllable data, where CELEX performed better. In the light of the number of datasets (four), the former result does not appear good reason for rejection with, for example, a Bonferroni corrected criterion of .0125. One result was significant at $5 \%$ but not $1.25 \%$ against the APEX model, and this was not with the corpus performing best for that data set.

\section{Contextual Diversity}

Why do many of these models of word frequency (WF) fail to capture the variability that appears to be due to word frequency? A serious problem for any attempt to account for these effects in this way arises because it appears that word frequency does not cause the effects that have been attributed to word frequency (irrespective of transformation). We have provided evidence elsewhere (Adelman et al., 2006) that a contextual diversity (CD) factor accounts for word frequency effects (cf. Galbraith \& Underwood, 1973; McDonald \& Shillcock, 2001). Since words tend to cluster in contexts, the likely need (Anderson \& Milson, 1989) of a word in an arbitrary new context relates to the number of contexts the word has been seen in before, not the number of occurrences of the word.

Documents are the natural contextual unit of a words in a corpus, and so we use this as a measurement of contextual diversity (cf. Steyvers \& Malmberg, 2003, who use this as a normative measure of context variability). With either logarithmic or power law transformations of WF and CD, Adelman et al. (2006) found a unique facilitatory influence of $\mathrm{CD}$, and the effects of WF were null or inhibitory, when assessed on mega-studies of lexical decision and word naming.

The possibility that $\mathrm{CD}$ rather than WF might be the relevant causal factor necessitates a re-evaluation of the models we have considered. The serial search model whence derives the rank hypothesis might yet be plausible if it is the rank of contextual diversity (and not the rank of word frequency) that determines the effects in question. 
Likewise, all of the other models may be modified by replacing word frequency with contextual diversity, with the exception of the duration-practice model, and the contexts model predicts the same functional form (exponential). Our list of functional forms is thus unaltered, and we can proceed as before.

Table 4 shows the $R^{2}$ values for contextual diversity fits to the various data sets with each of three corpora ${ }^{12}$. We note in passing that for the overwhelming majority $(117 / 120)$ of corpus-transformation combinations more variance was explained by CD than WF (consistent with the conclusions of Adelman et al., 2006). As before, most of the transformations performed better than rank, although the results with log CD are more mixed. Again, the bootstrap $\hat{p}$ values for the tests of the rank model in terms of the fit of the alternatives in Table 5 show that the rank model could be rejected on the basis that the alternative models would not fit so well were the rank model generating the data (82/108 at 5\% level, and 64/108 at $1.25 \%$ level, including every test for the Elexicon data and all but one test with TASA for the BCP99 young adult data).

Does the rank model account for variability that cannot be explained by the other models? Table 6 presents the results of the relevant bootstrap analyses (analogously to Table 3 but using CD in place of WF). There was evidence against the logarithmic transformation in 9 out of 12 combinations; against the power transformation in 8 out of 12 combinations, all 8 on mega-study data; against the race model on the Elexicon data with the two larger corpora; against the exponential transformation in all the analyses with Elexicon and the older adult data, as well as the TASA analysis on the BCP99 young adult data; against the IA1 model for the Elexicon-BNC combination; and against IA2 in 8 out of 12 combinations, all 8 on mega-study data. All of these models can be considered rejected.

The remaining three models, the generalized power model, the power exponential product (PEX) model and their common generalization, the APEX model, did not have 
any significant results against them at the Bonferroni-corrected criterion. Moreover, they appeared to account for a significant amount of variability in the data that the rank model did not. At this point however these three models have only faced the challenge of the rank model: It is possible that they do not capture all the variation captured by one of the other models. Table 7 presents the $\hat{p}$ values for the relevant tests; these tests were only performed using models that are not nested within the model that is under test. For reasons described above, we considered rejection at $\hat{p}<.0125$. Only one test met this criterion, testing the APEX model using the IA2 model on Elexicon, with the Kučera and Francis (1967) corpus, where BNC was the preferred corpus for this data set. Our analyses do not therefore provide strong evidence against any of the three remaining models.

\section{Discussion}

The aim of this paper was to investigate how the effects of word frequency can inform us about the processes involved in lexical decision in the light of recent attempts to account for such effects. To this end, we (i) described a method for assessing systematic divergences from model predictions that makes allowances for the parametric flexibility of models; (ii) analyzed the effect of the choice of frequency count to assess the effects; (iii) derived predictions of several models as to the form of the frequency effect; (iv) assessed the models against several existing data sets; and (v) investigated functional forms of the confounded factor of contextual diversity as a further explanation of effects attributed to word frequency.

There was evidence of systematic variability in lexical decision times that is systematically related to word frequency, but it cannot be explained by a linear relation with the rank of frequency. This variability may be related to contextual diversity, but rank contextual diversity does not account for all the variability due to contextual diversity. This undermines the case for serial search models of the type that Murray and 
Forster (2004) attempted to test, because these models' signature functional form of effect on response times was absent. Of course, some model in which serial search is only one of the components sensitive to frequency could be constructed; to test whether such a model is plausible, it would be necessary to specify the other processes and test that these processes alone do not account for the results, using some method that takes account of parametric complexity.

On the basis of our analyses, functional forms arising from extensions of instance models (Logan, 1988) that introduce background instances (generalized power), posit direct contextual access (PEX), or combine both of these features (APEX) remain viable for a basic functional form of contextual diversity effects in lexical decision. It remains to be seen whether these functional forms could be appropriate for response times to other tasks, and what functional relationships are predicted and are obtained from other measures such as dwell times.

Although these remaining functional forms are more flexible than several alternatives, the evidence was not within the range expected due to this flexibility alone. Nevertheless, should such functions remain viable, an important topic of research will be the theoretical interpretation of the parameters giving them their flexibility: There might be no such interpretation because the results could be artefactual of an approximation to some yet-to-be-suggested functional form, but the results might also reflect a general cognitive limit, or (perhaps more likely) individual differences between participants. Individual participant experiments like Murray and Forster's (2004) Experiment 3 are likely to be instructive as to the usefulness of these parameters: Of their three participants, two showed functional forms similar to a rank function, but one participant gave data similar to a log function; these differences may in fact be genuine individual parametric differences within one of these or another parameterized functional form, rather than noise. As this last possibility would imply difficulties in averaging over 
participants in the analysis of these models (see for instance, Heathcote et al., 2002), analysis at the level of individual participants will be necessary to test properly or otherwise explore models with nonlinear parameters (a rank model has no nonlinear parameters, although it is linear in neither word frequency nor contextual diversity).

Moreover, as with Murray and Forster's (2004) development, it is insufficient to posit a theory that accounts for only one effect in the data; Jacobs and Grainger (1994, p. 1316) suggest that "[m]any researchers would probably agree that a model that explains only one result or effect is not very interesting." A quantitative account of the source of the word frequency or contextual diversity effect in lexical decision does not account for lexical decision unless it can also capture other effects. The inclusion of covariates in our analyses is an imprecise proxy for a genuine account of these effects. Assessment of the quantitative ability of extensions of the models considered in this paper to account for other effects will likely also exclude them in their current form.

There is considerable scope for increased assessment of the quantitative fit of visual word recognition tasks. For such assessment to be useful, it must test the correct (null) hypothesis, that some proposed model is correct (not that two models are equivalent in fit) and disfavor overcomplexity without disfavoring necessary complexity, as well as accurately assessing the relevant variables. When such criteria are met, quantitative analyses can give important constraint and impetus to the modeling of visual word recognition, in this case including the rejection of the form of serial search model that Murray and Forster (2004) used to generate their predictions, and indications that instance models based on contextual diversity may explain effects attributed to word frequency. 


\section{Author note}

This work was supported by a Warwick Postgraduate Research Fellowship to the first author, and by grant RES 000-22-1558 from the Economic and Social Research Council (UK) and grant F/215/AY from the Leverhulme Trust. The authors are grateful to Neil Stewart and Elizabeth Maylor for discussions related to this work, José Quesada for calculating the TASA frequency and contextual diversity counts, and Elisabeth Blagrove for comments on an earlier version of the paper. 


\section{Footnotes}

${ }^{1}$ When differing numbers of linear parameters are involved, there is of course a well-known $R^{2}$-adjusted statistic for this. The correction has less influence when the number of points fitted is high; this illustrates an additional point to our later argument against the use of frequency bands.

${ }^{2}$ At least, the values of the predictor (vector) that are omitted from the experiment have been chosen a priori.

${ }^{3}$ One could almost equivalently use the parametric bootstrap that estimates the standard deviation of normal noise and then simulates on this basis. This would be similar to Murray and Forster's simulation procedure, except the standard deviation and other parameters would be based directly on the data.

${ }^{4}$ The result is not surprising relative to Murray and Forster's results: Their (high-variance) simulation had an average difference of .9\%, which is much smaller than the $3.7 \%$ yielded by the experiment.

${ }^{5}$ Ideally, using the rule $\hat{p}=\#\left(t^{*}>t\right) / B$ will give a distribution of $\hat{P}$ that is rectangular over $[0,1]$ when integrated over all possible data samples and estimated parameters to correctly reflect the tail probability of the evidence. To perform in effect an integration over the data and parameters in a finite fashion, instead of performing only a bootstrap on $T$ for the observed data, one can for each simulated data set perform a bootstrap on the basis of $P$. Roughly speaking, this places weight on likely data sets and hence likely parameters. One performs first a bootstrap sample, and calculates $\hat{p}$. $(1-\hat{p})$ now plays the role of $t$. Several more bootstrap replications are generated, and for each of these, $\hat{p}$ is calculated by estimating parameters and error distributions anew and simulating a $T$ distribution for each. The rank of the first level $\hat{p}$ among the second level $\hat{p}$ gives a second level $p$ estimate. If the $T$ chosen is not affected by parameter estimates, then the estimated distribution of $\hat{P}$ will be rectangular, and the two $p$ values will be the 
same.

${ }^{6}$ We use this portion of the corpus because these frequencies are more predictive of response times than those from the whole corpus. We attribute this to the overrepresentation of college-level texts in the full corpus for undergraduate level readers.

${ }^{7}$ One further mistranscription was noted when we conducted our analyses, and we used the true frequency of this item.

${ }^{8}$ For the BCP99 studies, these are from 2649, 2729, 2760 and 2774 items for Kučera-Francis, TASA, CELEX and BNC respectively. For Elexicon, the corresponding numbers are: 27053, 29461, 32838, 33991.

${ }^{9}$ Our results from the Kučera and Francis (1967) corpus for Murray and Forster's (2004) Experiment 1 were in the same direction as Murray and Forster's, but both correlations were smaller. This is not only due to the reduction in items and the use of undergraduate raters to adjust the ranks; the same discrepancy occurs with the data provided by Murray even when we used the ranks from the Appendix of their paper.

${ }^{10} \mathrm{An}$ example of one of these analyses in $\mathrm{R}$ code is given at http://www.warwick.ac.uk/ psrcaj/boot5p.R

${ }^{11}$ One of the non-significant tests is with the fit of a power law for Murray and Forster's (2004) Experiment 1 with Kučera and Francis (1967), which was a significant difference in our preliminary analysis with frequency bands above. The earlier analysis used the Kučera-Francis ranks estimates provided by Murray and Forster. These are less favorable to the rank hypothesis because they retain more words in the estimated vocabulary of undergraduates, which produces less of the distortive curvature illustrated in Figure 5. That is, removing a greater proportion of words in our rankings to reflect the empirical vocabulary of undergraduates exacerbates the problems of rank distortion from the incompleteness of the Kučera-Francis counts.

${ }^{12}$ CELEX was omitted because (i) its corpus was not available to us, and (ii) it is 
Modeling Lexical Decision 28

anyway formed of so few documents (contexts) that the CD measure could reasonably expected to perform poorly. (The documents do, however, contain many tokens.) 


\section{References}

Adelman, J. S., Brown, G. D. A., \& Quesada, J. F. (2006). Contextual diversity, not word frequency, determines word-naming and lexical decision times. Psychological Science, 17, 814-823.

Anderson, J. R., \& Milson, R. (1989). Human memory: An adaptive perspective. Psychological Review, 96, 703-719.

Andrews, S. (1989). Frequency and neighborhood effects on lexical access: Activation or search? Journal of Experimental Psychology: Learning, Memory, and Cognition, 15, 802-814.

Andrews, S. (1992). Frequency and neighborhood effects on lexical access: Lexical similarity or orthographic redundancy? Journal of Experimental Psychology: Learning, Memory, and Cognition, 18, 234-254.

Baayen, R. H., Piepenbrock, R., \& Gulikers, L. (1995). The CELEX Lexical Database (Release 2) [CD-ROM]. Philadelphia: Linguistic Data Consortium, University of Pennsylvania.

Balota, D. A., Cortese, M. J., Hutchison, K. A., Neely, J. H., Nelson, D., Simpson, G. B., et al. (2002). The English Lexicon Project: A web-based repository of descriptive and behavioral measures for 40,481 English words and nonwords. Retrieved 11th December, 2004 from http://elexicon.wustl.edu/ .

Balota, D. A., Cortese, M. J., \& Pilotti, M. (1999). Item-level analysis of lexical decision: Results from a mega-study. In Abstracts of the 40th Annual Meeting of the Psychonomics Society (p. 44). Los Angeles, CA: Psychonomic Society.

Balota, D. A., Cortese, M. J., Sergent-Marshall, S. D., Spieler, D. H., \& Yap, M. J. (2004). Visual word recognition of single-syllable words. Journal of Experimental Psychology: General, 133, 283-316. 
Balota, D. A., Yap, M. J., Cortese, M. J., Hutchison, K. I., Kessler, B., Loftis, B., et al. (2007). The English Lexicon Project. Behavior Research Methods, 39, 445-459.

Beran, R. (1988). Prepivoting test statistics: A bootstrap view of asymptotic refinements. Journal of the American Statistical Association, 83, 687-697.

British National Corpus Consortium. (2000). British National Corpus World Edition [CD-ROM]. Oxford: Humanities Computing Unit, University of Oxford.

Burgess, C., \& Livesay, K. (1998). The effect of corpus size in predicting reaction time in a basic word recognition task: Moving on from Kučera and Francis. Behavior Research Methods, Instruments and Computers, 30, 272-277.

Colonius, H. (1993). The instance theory of automaticity: Why the Weibull? Psychological Review, 102, 744-750.

Coltheart, M., Davelaar, E., Jonasson, J. T., \& Besner, D. (1977). Access to the internal lexicon. In S. Dornič (Ed.), Attention and performance VI (pp. 535-555). Hillsdale, NJ: Erlbaum.

Coltheart, M., Rastle, K., Perry, C., Langdon, R., \& Ziegler, J. (2001). DRC: A dual route cascaded model of visual word recognition and reading aloud. Psychological Review, 108, 204-256.

Efron, B., \& Tibshirani, R. J. (1993). An introduction to the bootstrap. New York: Chapman and Hall.

Galbraith, R. C., \& Underwood, B. J. (1973). Perceived frequency of concrete and abstract words. Memory $\& 3$ Cognition, 1, 56-60.

Heathcote, A., Brown, S., \& Mewhort, D. J. K. (2002). The power law repealed: The case for an exponential law of practice. Psychonomic Bulletin \& Review, 7, 185-207.

Jacobs, A. M., \& Grainger, J. (1994). Models of visual word recognition — sampling the 
state of the art. Journal of Experimental Psychology: Human Perception and Performance, 20, 1311-1334.

Kučera, H., \& Francis, W. N. (1967). Computational analysis of present-day American English. Providence, RI: Brown University Press.

Landauer, T. K., Foltz, P. W., \& Laham, D. (1998). An introduction to latent semantic analysis. Discourse Processes, 25, 259-284.

Logan, G. D. (1988). Toward an instance theory of automatization. Psychological Review, $95,492-527$.

McDonald, S. A., \& Shillcock, R. C. (2001). Rethinking the word frequency effect: The neglected role of distributional information in lexical processing. Language and Speech, 44, 295-323.

Murray, W. S., \& Forster, K. I. (2004). Serial mechanisms in lexical access: The rank hypothesis. Psychological Review, 111, 721-756.

Norris, D. (2006). The Bayesian reader: Explaining word recognition as an optimal Bayesian decision process. Psychological Review, 113, 327-357.

Steyvers, M., \& Malmberg, K. J. (2003). The effect of normative context variability on recognition memory. Journal of Experimental Psychology: Learning, Memory, and Cognition, 29, 760-766. 


\begin{tabular}{|c|c|c|c|c|c|c|c|c|c|c|c|}
\hline Data & Corpus & rank WF & log. WF & power WF & gen. pow. WF & race $\mathrm{WF}$ & exp. WF & APEX WF & PEX WF & IA1 WF & IA2 WF \\
\hline \multirow[t]{4}{*}{ MF Exp. 1} & Kučera-Francis & 21.13 & 20.35 & 21.62 & 21.62 & 20.93 & 19.69 & 21.65 & 21.65 & 21.40 & 21.68 \\
\hline & TASA & 18.96 & 20.76 & 20.90 & 22.14 & 22.13 & 21.59 & 22.14 & 21.66 & 22.13 & 20.88 \\
\hline & CELEX & 19.03 & 22.38 & 22.80 & 23.72 & 23.51 & 22.71 & 23.76 & 23.33 & 23.72 & 22.75 \\
\hline & $\mathrm{BNC}$ & 22.19 & 22.28 & 23.06 & 23.16 & 22.80 & 22.10 & 23.22 & 23.21 & 23.12 & 23.01 \\
\hline \multirow[t]{4}{*}{ BCP99 Young } & Kučera-Francis & 32.54 & 27.99 & 32.31 & 33.62 & 33.48 & 33.51 & 33.63 & 33.62 & 33.48 & 31.92 \\
\hline & TASA & 40.67 & 38.23 & 41.57 & 42.41 & 44.53 & 44.17 & 44.57 & 44.44 & 44.53 & 41.18 \\
\hline & CELEX & 35.55 & 35.11 & 38.49 & 42.80 & 42.67 & 42.49 & 42.80 & 42.55 & 42.67 & 37.99 \\
\hline & $\mathrm{BNC}$ & 36.27 & 33.19 & 35.72 & 39.73 & 39.68 & 39.36 & 39.74 & 39.46 & 39.68 & 35.32 \\
\hline \multirow[t]{4}{*}{ BCP99 Older } & Kučera-Francis & 27.80 & 23.20 & 27.69 & 27.80 & 27.76 & 27.04 & 27.81 & 27.80 & 27.81 & 27.50 \\
\hline & TASA & 34.90 & 31.42 & 35.10 & 35.57 & 36.08 & 35.13 & 36.16 & 35.94 & 36.16 & 34.77 \\
\hline & CELEX & 33.71 & 29.91 & 34.49 & 36.23 & 36.22 & 35.32 & 36.23 & 35.91 & 36.22 & 33.96 \\
\hline & $\mathrm{BNC}$ & 32.71 & 28.44 & 32.12 & 33.11 & 33.03 & 32.03 & 33.15 & 33.15 & 33.13 & 31.74 \\
\hline \multirow[t]{4}{*}{ Elexicon } & Kučera-Francis & 48.56 & 47.62 & 48.59 & 48.87 & 48.87 & 48.72 & 48.89 & 48.88 & 48.87 & 48.52 \\
\hline & TASA & 48.99 & 48.66 & 49.32 & 49.72 & 49.58 & 48.92 & 49.77 & 49.69 & 49.76 & 49.27 \\
\hline & CELEX & 48.72 & 50.78 & 51.03 & 51.80 & 51.75 & 51.26 & 51.87 & 51.82 & 51.84 & 51.01 \\
\hline & $\mathrm{BNC}$ & 51.10 & 51.71 & 51.76 & 52.63 & 52.52 & 51.92 & 52.71 & 52.62 & 52.70 & 51.76 \\
\hline
\end{tabular}




\begin{tabular}{|c|c|c|c|c|c|c|c|c|c|c|}
\hline \multirow[t]{3}{*}{ MF Exp. 1} & Kučera-Francis & .039 & .127 & .466 & .768 & .726 & .439 & .256 & .450 & .068 \\
\hline & TASA & .000 & .005 & .019 & .000 & .002 & .015 & .005 & .001 & .007 \\
\hline & CELEX & .000 & .000 & .000 & .000 & .000 & .000 & .000 & .000 & .000 \\
\hline \multirow[t]{3}{*}{ BCP99 Young } & Kučera-Francis & .052 & .707 & .038 & .000 & .000 & .003 & .000 & .000 & .802 \\
\hline & TASA & .000 & .000 & .000 & .000 & .000 & .001 & .000 & .000 & .000 \\
\hline & CELEX & .000 & .000 & .000 & .000 & .000 & .000 & .000 & .000 & .000 \\
\hline \multirow{3}{*}{ BCP99 Older } & TASA & .000 & .011 & .003 & .000 & .000 & .008 & .000 & .000 & .011 \\
\hline & CELEX & .000 & .001 & .000 & .000 & .000 & .000 & .000 & .000 & .000 \\
\hline & $\mathrm{BNC}$ & .003 & .248 & .013 & .001 & .000 & .064 & .001 & .005 & .120 \\
\hline \multirow[t]{4}{*}{ Elexicon } & Kučera-Francis & .000 & .008 & .000 & .000 & .000 & .000 & .000 & .000 & .043 \\
\hline & TASA & .000 & .000 & .000 & .000 & .000 & .000 & .000 & .000 & .000 \\
\hline & CELEX & .000 & .000 & .000 & .000 & .000 & .000 & .000 & .000 & .000 \\
\hline & $\mathrm{BNC}$ & .000 & .000 & .000 & .000 & .000 & .000 & .000 & .000 & .000 \\
\hline
\end{tabular}




\begin{tabular}{|c|c|c|c|c|c|c|c|c|c|c|}
\hline \multirow[t]{3}{*}{ MF Exp. 1} & Kučera-Francis & .001 & .491 & .301 & .040 & .001 & .311 & .400 & .146 & .531 \\
\hline & TASA & .106 & .283 & .593 & .770 & .210 & .391 & .365 & .760 & .208 \\
\hline & CELEX & .100 & .660 & .548 & .367 & .044 & .956 & .506 & .714 & .447 \\
\hline \multirow[t]{3}{*}{ BCP99 Young } & Kučera-Francis & .000 & .000 & .352 & .879 & .026 & .773 & .382 & .870 & .000 \\
\hline & TASA & .000 & .000 & .004 & .648 & .000 & .167 & .244 & .568 & .000 \\
\hline & CELEX & .000 & .060 & .639 & .998 & .108 & .316 & .658 & .994 & .000 \\
\hline \multirow{3}{*}{ BCP99 Older } & TASA & .000 & .000 & .028 & .035 & .000 & .234 & .053 & .421 & .000 \\
\hline & CELEX & .000 & .000 & .385 & .167 & .000 & .191 & .112 & .224 & .000 \\
\hline & $\mathrm{BNC}$ & .000 & .000 & .088 & .006 & .000 & .022 & .042 & .017 & .000 \\
\hline \multirow[t]{4}{*}{ Elexicon } & Kučera-Francis & .000 & .000 & .306 & .395 & .000 & .788 & .444 & .346 & .000 \\
\hline & TASA & .000 & .000 & .409 & .000 & .000 & .879 & .017 & .541 & .000 \\
\hline & CELEX & .000 & 1.000 & .206 & .000 & .000 & .443 & .644 & .012 & .996 \\
\hline & $\mathrm{BNC}$ & .000 & .000 & .047 & .000 & .000 & .478 & .005 & .000 & .000 \\
\hline
\end{tabular}




\begin{tabular}{|c|c|c|c|c|c|c|c|c|c|c|c|}
\hline Data & Corpus & rank CD & $\log . \mathrm{CD}$ & power $\mathrm{CD}$ & gen. pow. CD & race $\mathrm{CD}$ & exp. CD & APEX CD & PEX CD & IA1 CD & IA2 CD \\
\hline \multirow[t]{3}{*}{ MF Exp. 1} & Kučera-Francis & 21.17 & 20.48 & 21.73 & 21.73 & 21.40 & 20.57 & 21.76 & 21.76 & 21.65 & 21.74 \\
\hline & TASA & 19.94 & 21.18 & 21.43 & 21.92 & 22.65 & 22.02 & 22.67 & 22.08 & 22.65 & 21.40 \\
\hline & $\mathrm{BNC}$ & 21.91 & 23.04 & 23.07 & 23.11 & 22.97 & 22.63 & 23.25 & 23.25 & 23.19 & 23.07 \\
\hline \multirow[t]{3}{*}{ BCP99 Young } & Kučera-Francis & 34.49 & 30.45 & 34.29 & 35.55 & 35.24 & 35.52 & 35.55 & 35.54 & 35.24 & 33.97 \\
\hline & TASA & 42.75 & 39.06 & 42.87 & 45.98 & 45.87 & 45.71 & 45.98 & 45.91 & 45.87 & 42.42 \\
\hline & $\mathrm{BNC}$ & 37.78 & 38.48 & 38.51 & 41.14 & 40.81 & 41.11 & 41.11 & 41.11 & 40.81 & 38.51 \\
\hline \multirow[t]{3}{*}{ BCP99 Older } & Kučera-Francis & 29.70 & 25.13 & 29.61 & 29.75 & 29.75 & 29.36 & 29.75 & 29.71 & 29.75 & 29.43 \\
\hline & TASA & 36.52 & 31.90 & 36.23 & 37.28 & 37.24 & 36.33 & 37.28 & 37.11 & 37.29 & 35.85 \\
\hline & $\mathrm{BNC}$ & 34.27 & 33.26 & 33.94 & 34.73 & 34.71 & 34.43 & 34.91 & 34.91 & 34.71 & 33.90 \\
\hline \multirow[t]{3}{*}{ Elexicon } & Kučera-Francis & 49.24 & 48.47 & 49.30 & 49.63 & 49.59 & 49.57 & 49.63 & 49.62 & 49.59 & 49.25 \\
\hline & TASA & 49.78 & 49.25 & 50.00 & 50.45 & 50.36 & 49.77 & 50.48 & 50.38 & 50.47 & 49.94 \\
\hline & $\mathrm{BNC}$ & 51.54 & 52.60 & 52.60 & 53.13 & 53.07 & 52.69 & 53.20 & 53.17 & 53.16 & 52.60 \\
\hline
\end{tabular}




\begin{tabular}{|c|c|c|c|c|c|c|c|c|c|c|}
\hline Data & Corpus & log. CD & power $\mathrm{CD}$ & gen. pow. CD & race $\mathrm{CD}$ & exp. CD & APEX CD & PEX CD & IA1 CD & IA2 CD \\
\hline \multirow[t]{3}{*}{ MF Exp. 1} & Kučera-Francis & .040 & .081 & .385 & .426 & .442 & .326 & .171 & .227 & .078 \\
\hline & TASA & .000 & .017 & .017 & .000 & .002 & .027 & .014 & .000 & .016 \\
\hline & $\mathrm{BNC}$ & .000 & .025 & .006 & .066 & .033 & .017 & .036 & .022 & .021 \\
\hline \multirow[t]{3}{*}{ BCP99 Young } & Kučera-Francis & .079 & .810 & .048 & .000 & .000 & .002 & .000 & .000 & .897 \\
\hline & TASA & .000 & .011 & .001 & .000 & .000 & .001 & .000 & .000 & .014 \\
\hline & $\mathrm{BNC}$ & .000 & .000 & .000 & .000 & .000 & .000 & .000 & .000 & .000 \\
\hline \multirow[t]{3}{*}{ BCP99 Older } & Kučera-Francis & .673 & .844 & .382 & .160 & .128 & .356 & .791 & .248 & .827 \\
\hline & TASA & .001 & .343 & .019 & .001 & .000 & .047 & .003 & .000 & .308 \\
\hline & $\mathrm{BNC}$ & .005 & .340 & .065 & .001 & .000 & .036 & .001 & .027 & .267 \\
\hline \multirow[t]{3}{*}{ Elexicon } & Kučera-Francis & .000 & .002 & .001 & .000 & .000 & .000 & .000 & .000 & .011 \\
\hline & TASA & .000 & .000 & .000 & .000 & .000 & .000 & .000 & .000 & .000 \\
\hline & $\mathrm{BNC}$ & .000 & .000 & .000 & .000 & .000 & .000 & .000 & .000 & .000 \\
\hline
\end{tabular}

Note - Values here are based on 999 bootstrap 'replications'. 


\begin{tabular}{|c|c|c|c|c|c|c|c|c|c|c|}
\hline Data & Corpus & log. CD & power CD & gen. pow. CD & race $\mathrm{CD}$ & exp. CD & APEX CD & PEX CD & IA1 CD & IA $2 \mathrm{CD}$ \\
\hline \multirow[t]{3}{*}{ MF Exp. 1} & Kučera-Francis & .003 & .533 & .302 & .103 & .008 & .345 & .434 & .325 & .481 \\
\hline & TASA & .064 & .197 & .502 & .839 & .229 & .443 & .292 & .857 & .143 \\
\hline & $\mathrm{BNC}$ & .277 & .383 & .524 & .171 & .072 & .566 & .396 & .519 & .376 \\
\hline \multirow[t]{3}{*}{ BCP99 Young } & Kučera-Francis & .000 & .002 & .426 & .979 & .178 & .842 & .467 & .967 & .000 \\
\hline & TASA & .000 & .000 & .173 & .853 & .001 & .228 & .268 & .827 & .000 \\
\hline & $\mathrm{BNC}$ & .000 & .000 & .574 & .985 & .336 & .428 & .441 & .991 & .000 \\
\hline \multirow[t]{3}{*}{ BCP99 Older } & Kučera-Francis & .000 & .009 & .266 & .446 & .000 & .247 & .073 & .414 & .002 \\
\hline & TASA & .000 & .000 & .393 & .082 & .000 & .231 & .033 & .428 & .000 \\
\hline & $\mathrm{BNC}$ & .000 & .000 & .133 & .021 & .000 & .129 & .225 & .012 & .000 \\
\hline \multirow[t]{3}{*}{ Elexicon } & Kučera-Francis & .000 & .000 & .487 & .981 & .000 & .558 & .493 & .985 & .000 \\
\hline & TASA & .000 & .000 & .276 & .000 & .000 & .502 & .016 & .539 & .000 \\
\hline & $\mathrm{BNC}$ & 1.000 & 1.000 & .163 & .000 & .000 & .512 & .193 & .000 & 1.000 \\
\hline
\end{tabular}

Note - Values here are based on 999 bootstrap 'replications'. 


\begin{tabular}{|c|c|c|c|c|c|c|c|c|c|c|c|c|c|}
\hline Data & \multirow{2}{*}{$\begin{array}{l}H_{0}: \\
H_{a}:\end{array}$} & \multicolumn{5}{|c|}{ gen. pow. $\mathrm{CD}$} & \multicolumn{5}{|c|}{ PEX CD } & \multicolumn{2}{|c|}{ APEX CD } \\
\hline Corpus & & exp. CD & APEX CD & PEX CD & IA1 CD & IA2 CD & race $\mathrm{CD}$ & gen. pow. CD & APEX CD & IA1 CD & IA2 CD & IA1 CD & IA2 CD \\
\hline \multicolumn{14}{|l|}{ MF Exp. 1} \\
\hline Kučera-Fran & & .333 & .549 & .743 & .561 & .618 & .522 & .458 & .193 & .547 & .665 & .642 & .730 \\
\hline TASA & & .038 & .441 & .273 & .498 & .117 & .129 & .998 & .994 & .999 & .948 & .715 & .141 \\
\hline $\mathrm{BNC}$ & & .758 & .597 & .651 & .958 & .619 & .472 & .497 & .603 & .551 & .589 & .720 & .697 \\
\hline \multicolumn{14}{|l|}{ BCP99 Young } \\
\hline Kučera-Fran & & .573 & .489 & .751 & .389 & .364 & .476 & .730 & .796 & .544 & .425 & .451 & .383 \\
\hline TASA & & .681 & .561 & .545 & .491 & .490 & .330 & .969 & .894 & .975 & .829 & .492 & .516 \\
\hline $\mathrm{BNC}$ & & .312 & .146 & .435 & .336 & .189 & .283 & .966 & .863 & .821 & .591 & .838 & .590 \\
\hline \multicolumn{14}{|l|}{ BCP99 Older } \\
\hline Kučera-Fran & & .529 & .288 & .294 & .766 & .598 & .605 & .906 & .892 & .945 & .805 & .928 & .626 \\
\hline TASA & & .597 & .041 & .676 & .713 & .686 & .315 & .995 & .970 & .999 & .992 & .908 & .446 \\
\hline $\mathrm{BNC}$ & & 1.000 & .981 & .624 & .568 & .997 & .909 & .627 & .420 & .599 & .792 & .681 & .851 \\
\hline \multicolumn{14}{|l|}{ Elexicon } \\
\hline Kučera-Fran & & .565 & .978 & 1.000 & .167 & .016 & .449 & .985 & .997 & .814 & .122 & .036 & .007 \\
\hline TASA & & .859 & 1.000 & .739 & 1.000 & .245 & .080 & 1.000 & 1.000 & 1.000 & .984 & .078 & .045 \\
\hline $\mathrm{BNC}$ & & .999 & .999 & .985 & 1.000 & 1.000 & .039 & 1.000 & 1.000 & 1.000 & .967 & .997 & .981 \\
\hline
\end{tabular}




\section{Figure Captions}

Figure 1. Estimated distribution of $R^{2}(\%)$ for power law fit to rank hypothesis data, Murray and Forster's (2004) Experiment 1, using condition means. Number of bootstrap samples, $B=10,001$. Vertical line is observed $R^{2}$ for power law fit.

Figure 2. Estimated distribution of difference in $R^{2}(\%)$ between power law and rank fits to rank hypothesis data, Murray and Forster's (2004) Experiment 1, using condition means. Number of bootstrap samples, $B=10,001$. Vertical line is observed difference.

Figure 3. Estimated distribution of Hotelling $T$ statistic comparing power law and rank fits to rank hypothesis data, Murray and Forster's (2004) Experiment 1, using condition means. Number of bootstrap samples, $B=10,001$. Vertical line is observed difference.

Figure 4. Estimated distribution of Hotelling $T$ statistic comparing rank and power law fits to power law hypothesis data, Murray and Forster's (2004) Experiment 1, using condition means. Number of bootstrap samples, $B=10,001$. Vertical line is observed difference.

Figure 5. Relationship between corpus estimates of frequency. (a) Comparisons involving rank word frequency. (b) Comparisons involving log. word frequency. Whilst the logarithmic transformation of frequency is approximately linearly related between corpora, the relationship between ranks estimated from different corpora can be far from linear. As these ranks were adjusted to accord with undergraduate vocabulary (size), the differences must come from the incompleteness of some corpora. 


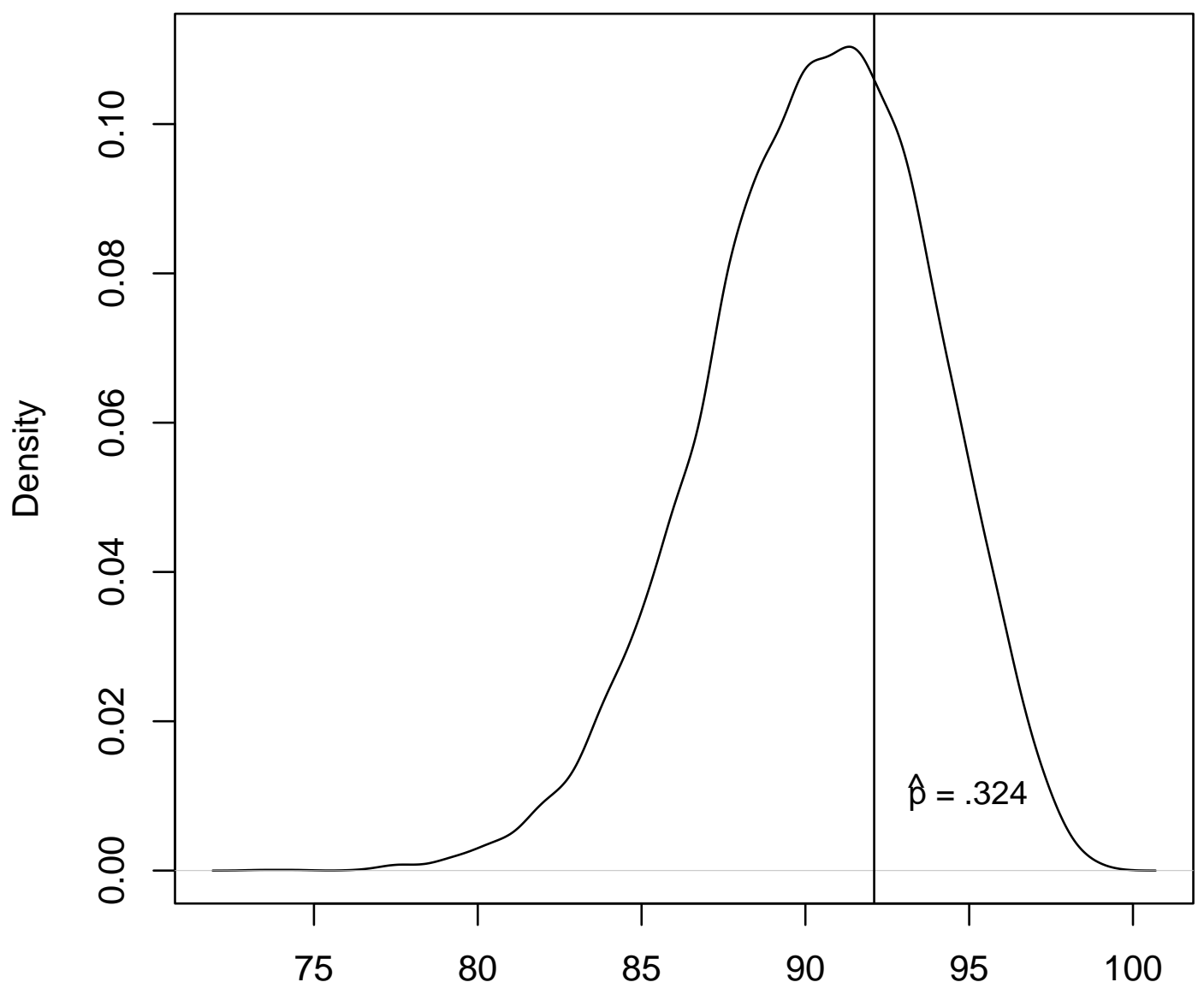

Variance Accounted for (\%) by Power Law of Frequency 


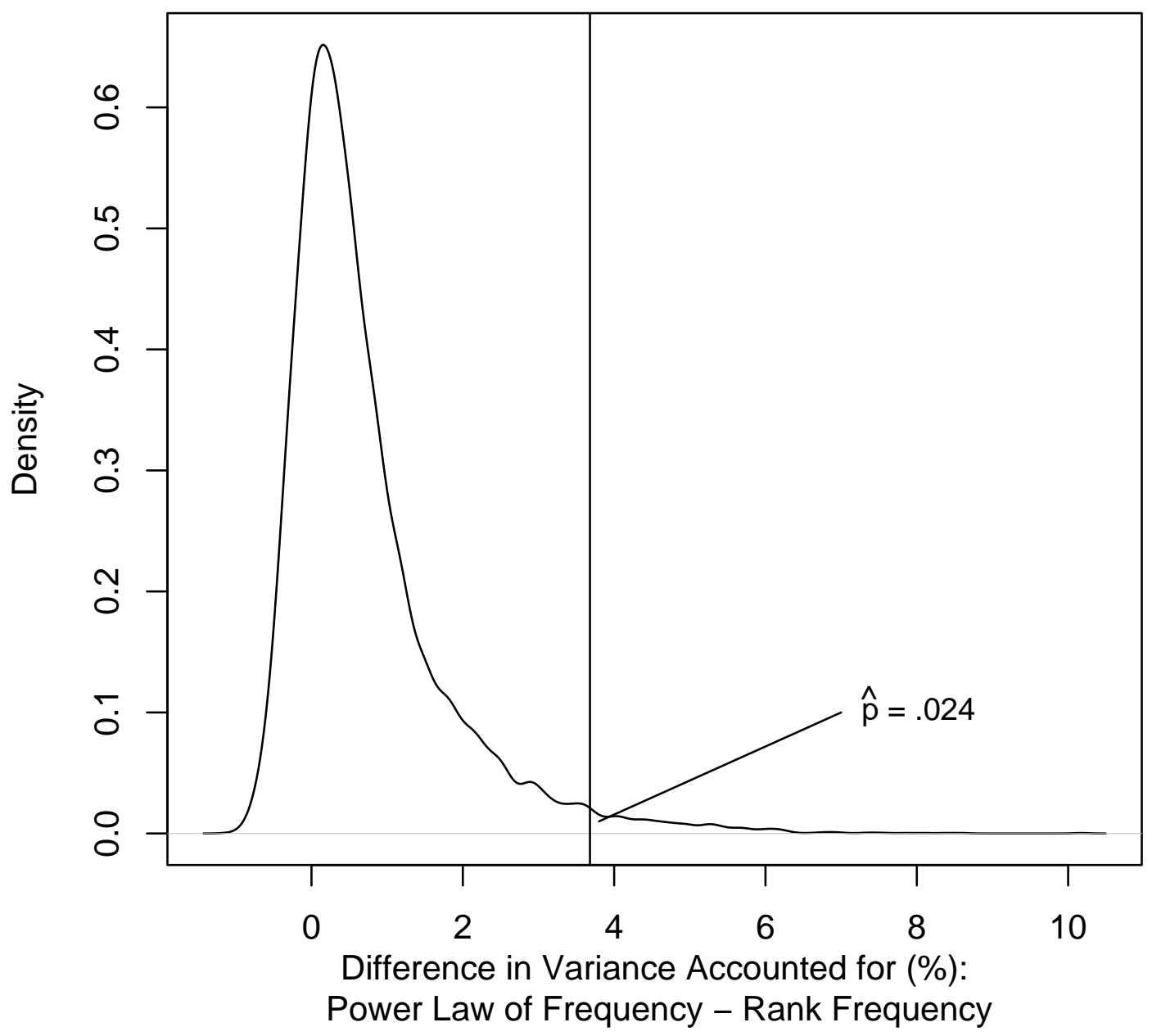




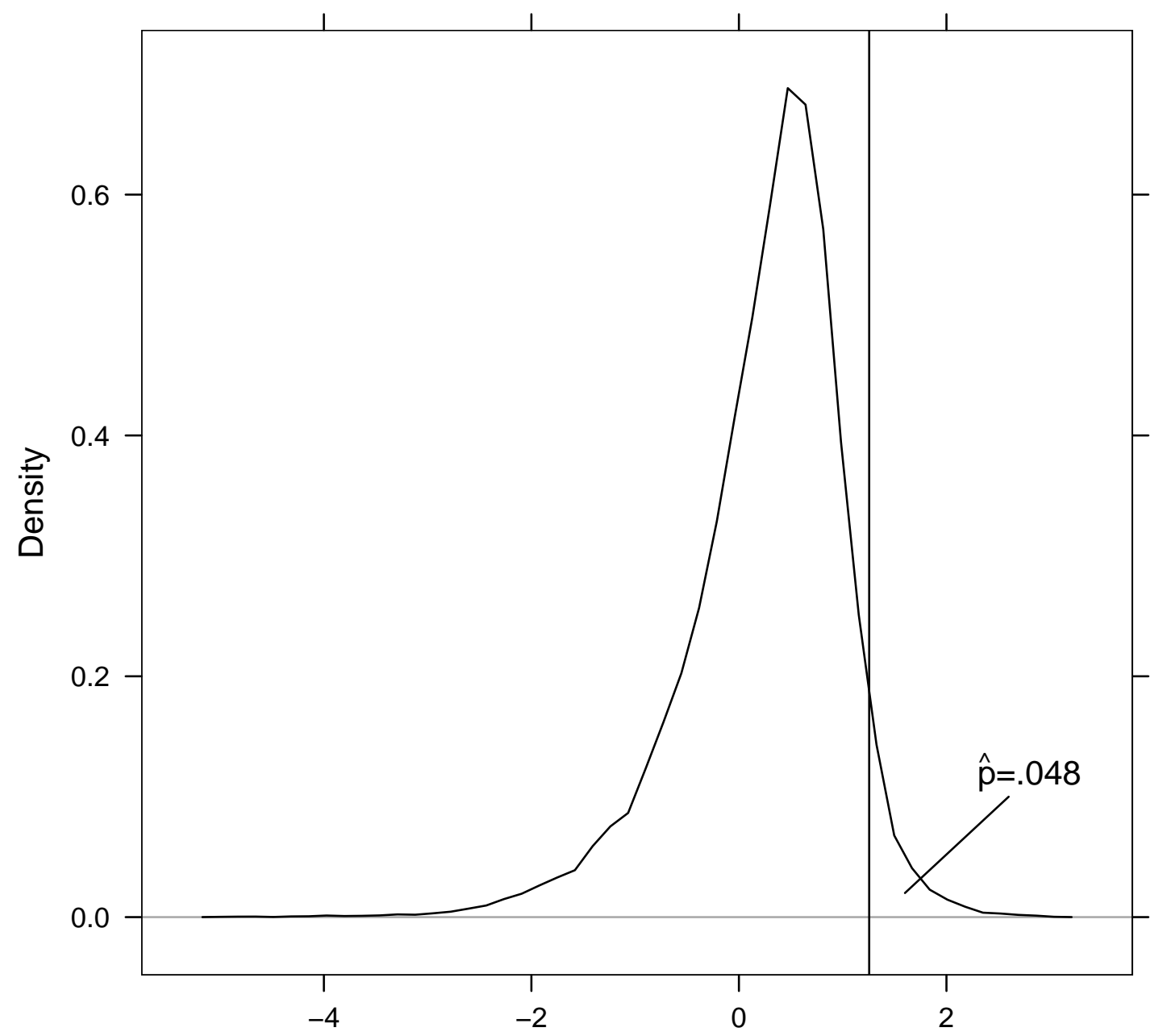

Hotelling Statistic:

Power Law of Frequency vs. Rank Frequency 


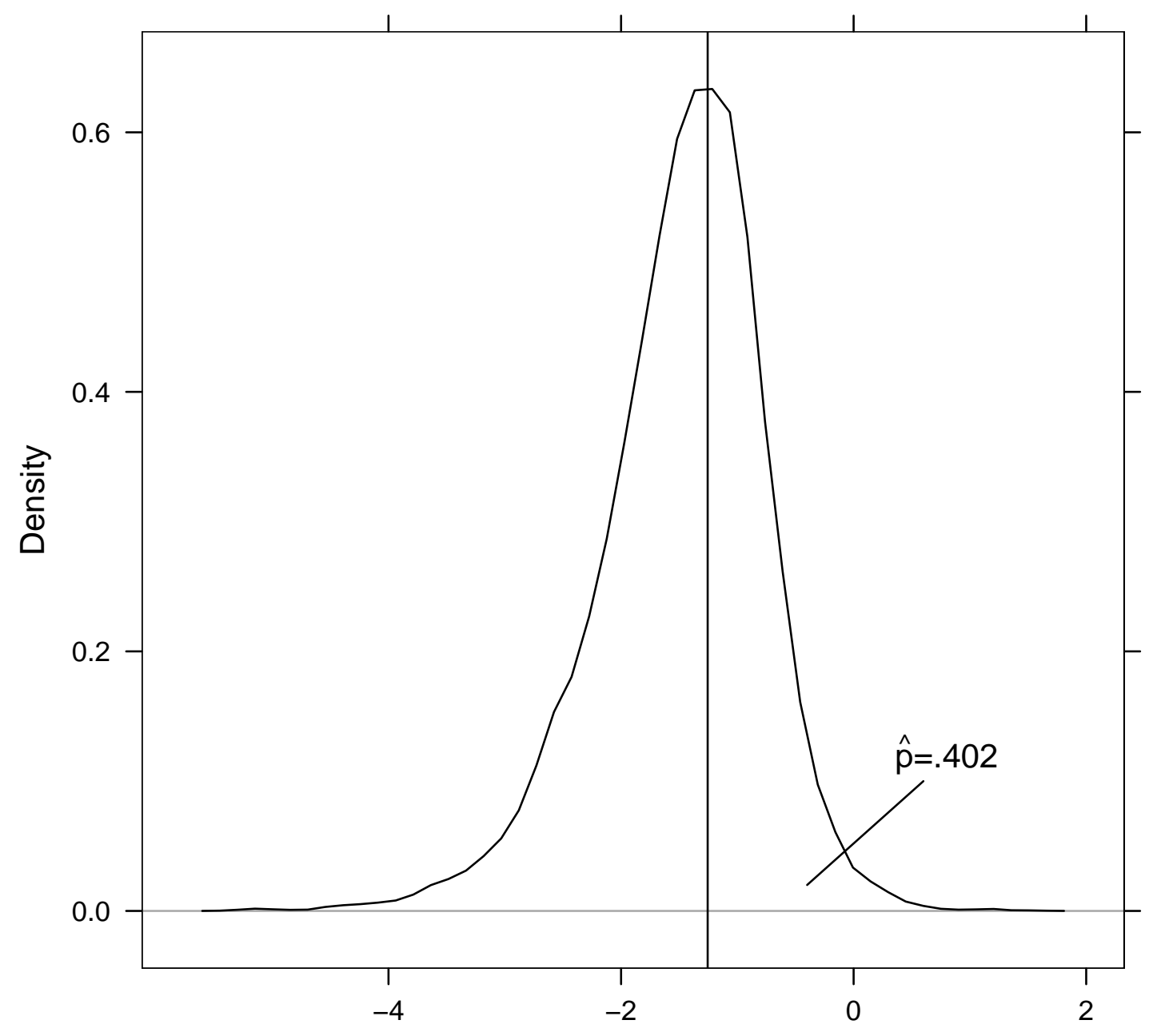

Hotelling Statistic:

Rank Frequency vs. Power Law of Frequency 


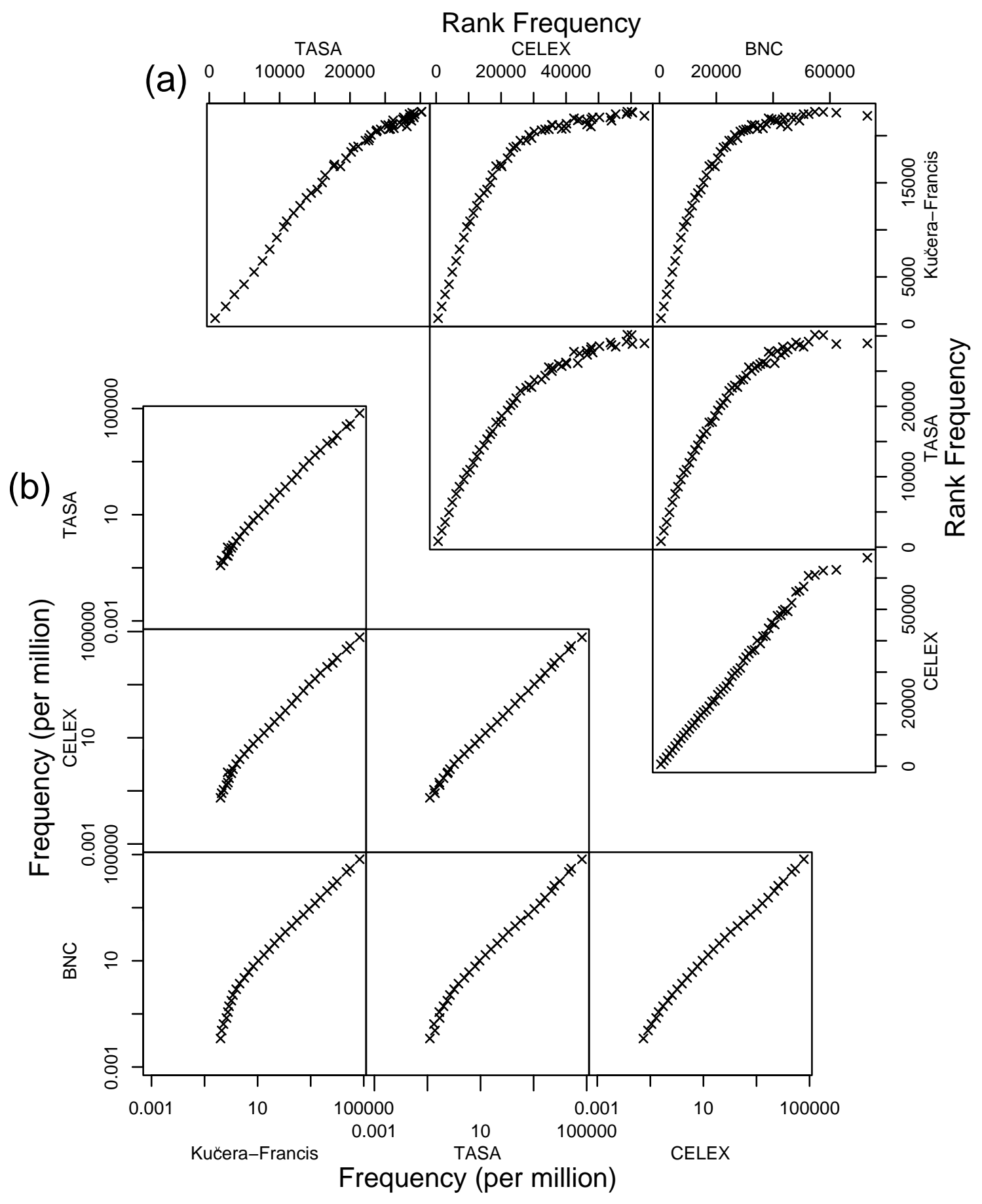


Postscript: Deviations from the Predictions of Serial Search

James S. Adelman and Gordon D. A. Brown

University of Warwick 
Murray and Forster (2004) claimed that rank frequency provided a better account of lexical decision times than either log frequency or power law frequency, the latter being dismissed on the grounds of over-flexibility. We (Adelman \& Brown, 2008) argued that (i) Murray and Forster's use of the relatively small Kučera and Francis (1967) word frequency counts biased the estimates of rank; (ii) the superiority in fit of the power law (and of some other functions) could not all be attributed to over-flexibility in the manner Murray and Forster claimed; and (iii) bootstrapping analyses designed to take flexibility into account gave evidence of systematic deviations from several theoretically-motivated functional forms, including rank and power, but not from some generalizations of the power function. We concluded that the data could not be taken as support for serial search models.

Murray and Forster (2008) have suggested that our results do not contradict the rank hypothesis (and in fact support it) because (i) an additional task-specific mechanism could account for any discrepancy between data and model predictions; (ii) the increase in $R^{2}$ for rank when better-estimated ranks are used provides stronger evidence for rank, and shows that the case for rank was not favored by a bias in rank estimates; (iii) Adelman and Brown (2008) did not find a systematic failure of the rank function; (iv) assessment of the rank function does not rely on estimation of parameters, but assessment of the power function relies on parameters that lack theoretical interpretation or independent justification, unlike those of the rank function; (v) their simulations of an instance model show that such models cannot provide a plausible account of mean lexical decision latencies; and (vi) data from different tasks, data sets, and measures converge in favor of the rank function.

In response to Murray and Forster's (2008) comments, we make the following points. (i) An appeal to additional mechanisms can of course be made for any theory, but - absent a detailed specification and test of such mechanisms — such appeal inevitably raises serious concerns about unfalsifiability. (ii) All the models, not just the rank model, 
show an increase in $R^{2}$ with the move to different corpora. This reflects less noisy frequency estimates, and for the mega-study data, the inclusion of more lower-frequency words, which leads to a broader frequency range. Therefore, such increases cannot be taken as better evidence for rank. Moreover, such increases could mask any underlying disadvantage to rank given by the use of more accurate ranks. The empirical evidence still shows that the use of Kučera and Francis (1967) gives systematic underestimates of the rank of low frequency words. (iii) We do not of course dispute the claim that there is a significant correlation between rank frequency and lexical processing time, nor do we deny the problems associated with a logarithmic function. However, we argued that the improvement in fit shown by several other functions is significantly greater than would be the case were the rank hypothesis correct. Indeed, of 144 possible tests of the rank hypothesis in our Table 2, 117 gave evidence against it. Murray and Forster (2008) correctly note variability of the $\hat{p}$ values, but perfect repetition of $p$-values is not to be expected, and very high values were only observed with the (suspect) Kučera and Francis (1967) ranks. (iv) The parameters of the rank function may be interpreted within serial search as the time for processes other than the serial search (intercept), and time taken to search each word (slope), neither of which can be inferred without reference to data. Furthermore, the sidetrack model has a non-linear mantissa parameter that was fixed by Murray and Forster (2004) with reference to the data. Regarding theoretical interpretation for the exponent in a power law (or more complex functions containing a power law), we noted that the exponent in a power function from the model we described can be related to the rate of increase in accessibility of traces once a word has been presented (Colonius, 1993). In any case, we suggest it would be premature to dismiss functions that require exponents, if such functions fit the data better than rank frequency, just on the grounds that independent justification for the parameter values is not yet available. (v) We entirely agree with Murray and Forster (2008) that the instance model 
they tested cannot be correct. Indeed, such a model is known to give results equivalent to the plain power law, and we provided evidence against the fit of such a function ourselves. We also note that use of a symmetrical distribution for trace retrieval implies a symmetrical distribution for response times in the model, contrary to that typically observed. Indeed, a further argument against the plain power law within the instance framework we described is that it gives left-skewed response time distributions when the exponent is in the range seen in the data; the same criticism does not apply to the generalized power laws that are also consistent with the mean latencies. (vi) Whilst presenting a model that also makes a prediction for error rates is laudable, it seems clear that a core prediction of the account concerns latencies, including those in lexical decision. Any systematic deviation from this prediction must therefore be viewed as problematic for the model irrespective of its other performance, and we now argue that a similar deviation may be observed across a variety of sources of latencies.

Figure 1 illustrates the last point with the Kučera and Francis (1967) ranks used by Murray and Forster $(2004,2008)$. Lexical decision, word naming and eye movement data all tend to show overprediction by rank for extreme ranks and underprediction for intermediate ones. The sole exception is for the (somewhat rarely used) "sum of first three fixations" measure from Murray (2001). Virtuously, the serial search model espoused by Murray and Forster makes a strong, testable prediction. The systematic and task-independent discrepancy between model predictions and data suggests to us - in the absence of an extended model demonstrated to rectify the discrepancy - that the case for serial search has yet to be adequately made. 


\section{Author Note}

This work was supported by grant RES 062-23-0545 from the Economic and Social Research Council (UK). 


\section{References}

Adelman, J. S., \& Brown, G. D. A. (2008). Modeling lexical decision: The form of frequency and diversity effects. Psychological Review.

Balota, D. A., Cortese, M. J., Hutchison, K. A., Neely, J. H., Nelson, D., Simpson, G. B., et al. (2002). The English Lexicon Project: A web-based repository of descriptive and behavioral measures for 40,481 English words and nonwords. Retrieved 11th December, 2004 from http://elexicon.wustl.edu/ .

Balota, D. A., \& Spieler, D. H. (1998). The utility of item-level analyses in model evaluation: A reply to Seidenberg and Plaut. Psychological Science, 9, 238-240.

Colonius, H. (1993). The instance theory of automaticity: Why the Weibull? Psychological Review, 102, 744-750.

Kučera, H., \& Francis, W. N. (1967). Computational analysis of present-day American English. Providence, RI: Brown University Press.

Murray, W. S., \& Forster, K. I. (2004). Serial mechanisms in lexical access: The rank hypothesis. Psychological Review, 111, 721-756.

Murray, W. S., \& Forster, K. I. (2008). The rank hypothesis and lexical decision: A reply to Adelman and Brown (2008). Psychological Review.

Seidenberg, M. S., \& Waters, G. S. (1989). Word recognition and naming: A mega study. Bulletin of the Psychonomic Society, 27, 489.

Spieler, D. H., \& Balota, D. A. (1997). Bringing computational models of word naming down to the item level. Psychological Science, 8, 411-416.

Treiman, R., Mullennix, J., Bijeljac-Babic, R., \& Richmond-Welty, E. D. (1995). The special role of rimes in the description, use, and acquisition of English orthography. Journal of Experimental Psychology: General, 124, 107-136. 


\section{Figure Caption}

Figure 1. Discrepancy between data and predictions of rank model (loess smoothed plots, $\alpha=.8)$. Solid lines: lexical decision latences; dashed lines: word naming latencies; dot-dash lines: eye-movement study fixation/gaze durations. Mega-study data: (a) Seidenberg and Waters (1989) naming; (b) Spieler and Balota (1997) young adults naming; (c) Balota and Spieler (1998) older adults naming; (d) Balota et al. (2002) Elexicon naming; (e) Treiman, Mullennix, Bijeljac-Babic, and Richmond-Welty (1995) naming; (f) Balota et al. (2002) Elexicon LDT; (g) Balota et al. (1999) older adults LDT; and (h) Balota et al. (1999) young adults LDT. Murray and Forster's data: (i) Murray (2001) sum of 3 fixations; (j) Murray and Forster (2004) Exp. 3 KF; (k) Murray (2007) first fixation; (l) Murray and Forster (2004) Exp. 3 GT; (m) Murray (2007) last fixation; (n) Murray (2001) first fixation; (o) Murray and Forster (2004) Exp. 2; (p) Murray and Forster (2008) 1LD-foil LDT; (q) Murray and Forster (2008) DIS-foil LDT; (r) Murray and Forster (2004) Exp. 1; (s) Murray and Forster (2004) Exp. 3 WM; and (t) Murray (2007) gaze. 


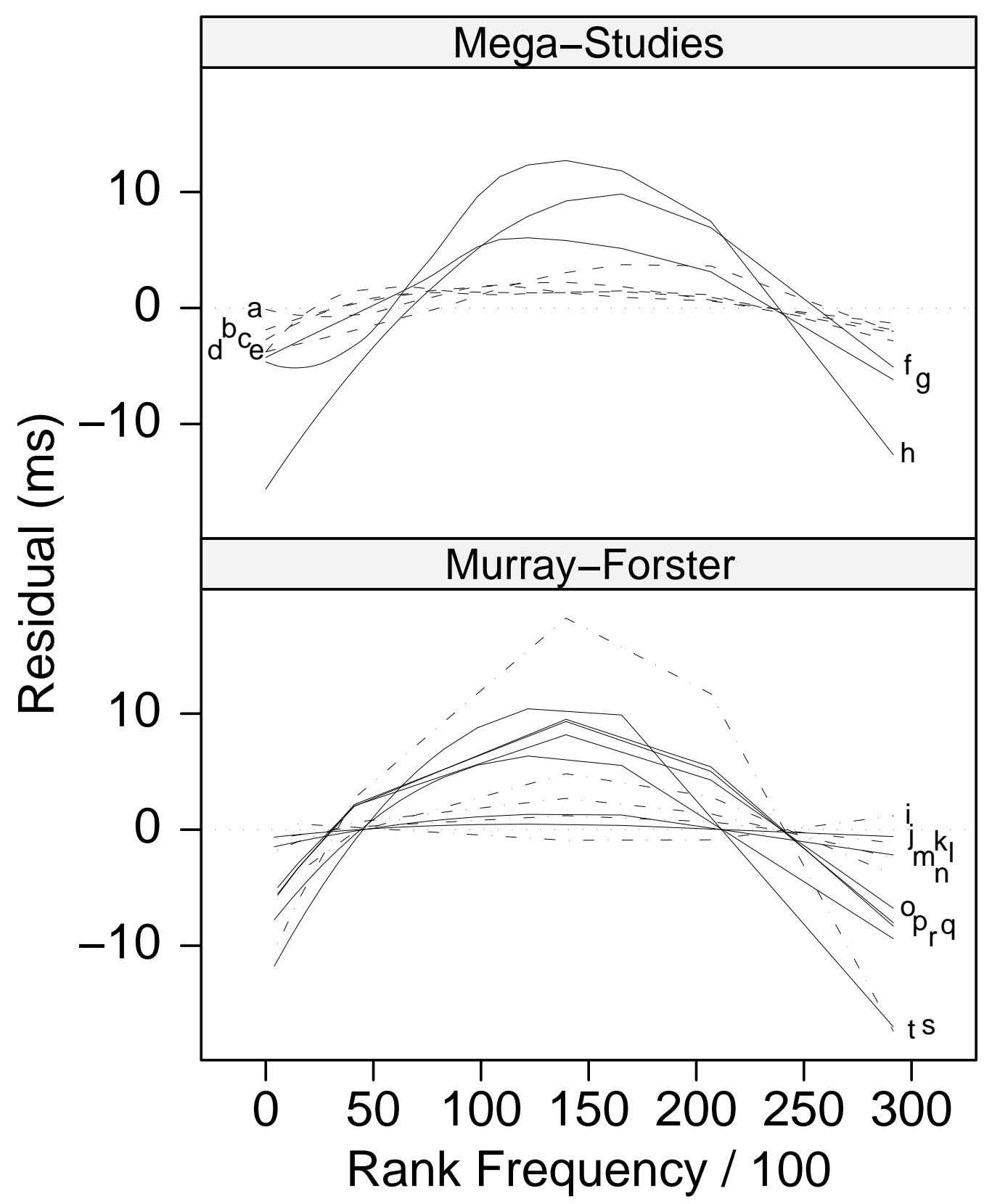

